\title{
Investigation of SINQ (Lead/Zircaloy) Spallation Target Structures by Means of Neutron Imaging Techniques
}

\author{
M. Wohlmuter ${ }^{2}$, S. Dementjevs ${ }^{2}$, P. Vontobel $^{1}$, J. Hovind ${ }^{1}$, P. Trtik ${ }^{1}$, \\ E.H. Lehmann ${ }^{1, a, *}$ \\ ${ }^{1}$ Laboratory for Neutron Scattering \& Imaging, Paul Scherrer Institut, CH-5232 Villigen PSI \\ ${ }^{2}$ Large Research Facilities Division, Paul Scherrer Institut, $\mathrm{CH}-5232$ Villigen PSI \\ aeberhard.lehmann@psi.ch \\ ${ }^{*}$ corresponding author
}

Keywords: Spallation Neutron Source, Target Technology, Melting Point Lead, Neutron Imaging, Gamma Dose Rate, Dy Converter Technique, Imaging Plates, NEURAP

\begin{abstract}
We report here on neutron imaging material studies of lead/zircaloy spallation target structures that are used to operate the Swiss Spallation Neutron Source (SINQ) facility at Paul Scherrer Institut (PSI). Lead provides a relatively high yield when exposed by the high intense proton beam delivered by the $590 \mathrm{MeV}$ cyclotron [1]. With the melting point of $327.5^{\circ} \mathrm{C}$, this target material gets liquid inside the Zircaloy cladding during the proton beam exposure of about 1 MW thermal power. Short beam interruptions and other shut-down phases result in the solidification of the lead structures. In order to investigate the long-term behavior of this relevant material during proton exposure methods of neutron imaging have been used: (i) on-line studies in thermal cycling time sequences during external heating of dummy rods and (ii) inspection of "spent" rods after dismounting from the rod bundle. While the first type of studies can be performed in a normal neutron imaging setup, the second kind of investigations needs a wellshielded configuration (NEURAP) together with an imaging method, insensitive to the accompanying gamma background from the highly activated rod samples.
\end{abstract}

\section{Introduction}

The Paul Scherrer Institut (PSI, Switzerland) successfully operates the spallation neutron source SINQ [2] since 1997. It is the most powerful national neutron source in Switzerland and the basis for neutron research, also accessible by the international user community via semiannual open calls for scientific proposals.

Lead was found the most useful material for the spallation process with its high neutron yield and the low neutron absorption cross section. The SINQ targets consists today of $\mathrm{Pb}$ filled Zircaloy-2 tubes of about $10 \mathrm{~mm}$ diameter and $20 \mathrm{~cm}$ length arranged in a hexagonal rod bundle, placed perpendicular to the proton beam direction. Colloquially, the Pb-filled Zircaloy-2 tubes are called "Cannellonis".

The "Cannellonis" bundle is cooled via a forced cross flow of heavy water $\left(\mathrm{D}_{2} \mathrm{O}\right)$. At the highest power level, the target operation has to allow for local melting of the $\mathrm{Pb}$ inside the Zicaloy-2 cladding rod. In cases of proton beam trips or other shutdown of the heating power the $\mathrm{Pb}$ freezes and starts shrinking immediately. For these cases of this thermal cycling a reduced filling of the Cannelloni's by $10 \%$ is foreseen during the configuration process.

In the last years, SINQ has been operated in competitive mode with another spallation target station - ultra cold neutron (UCN) source [3]; the full proton beam power is diverted to the UCN source for a few seconds only with a duty cycle of up to $3 \%$. This short period of missing beam 
power is enough to result in solidification of the molten $\mathrm{Pb}$ for the time of the beam kick to the UCN source.

The consequences for the target reliability have been studied in different manners. Next to thermo-dynamic simulations we performed two kinds of neutron imaging studies: in-situ studies for the melting/freezing process with heating/cooling elements and a mock-up target rod setup [2]; inspection of samples from used targets with the NEURAP technique [4]. Neutron imaging is a very useful approach since the target materials $(\mathrm{Pb}, \mathrm{Zr})$ are very transparent whereas common X-ray studies would fail due to the limited transmission. This report describes the applied techniques and shows preliminary results of selected examples.

\section{The spallation neutron source SINQ and its target technology}

Based on the already existing high intense proton accelerator at PSI, the spallation neutron source SINQ (Fig. 1) went into operation in 1997. Protons with $590 \mathrm{MeV}$ and a current of up to 2 $\mathrm{mA}$ are sent to the target where the conversion to neutrons happens by the spallation process in the lead target. Because the heating goes beyond the melting point of lead, the Zircaloy cladding provides the real barrier during high power operation.
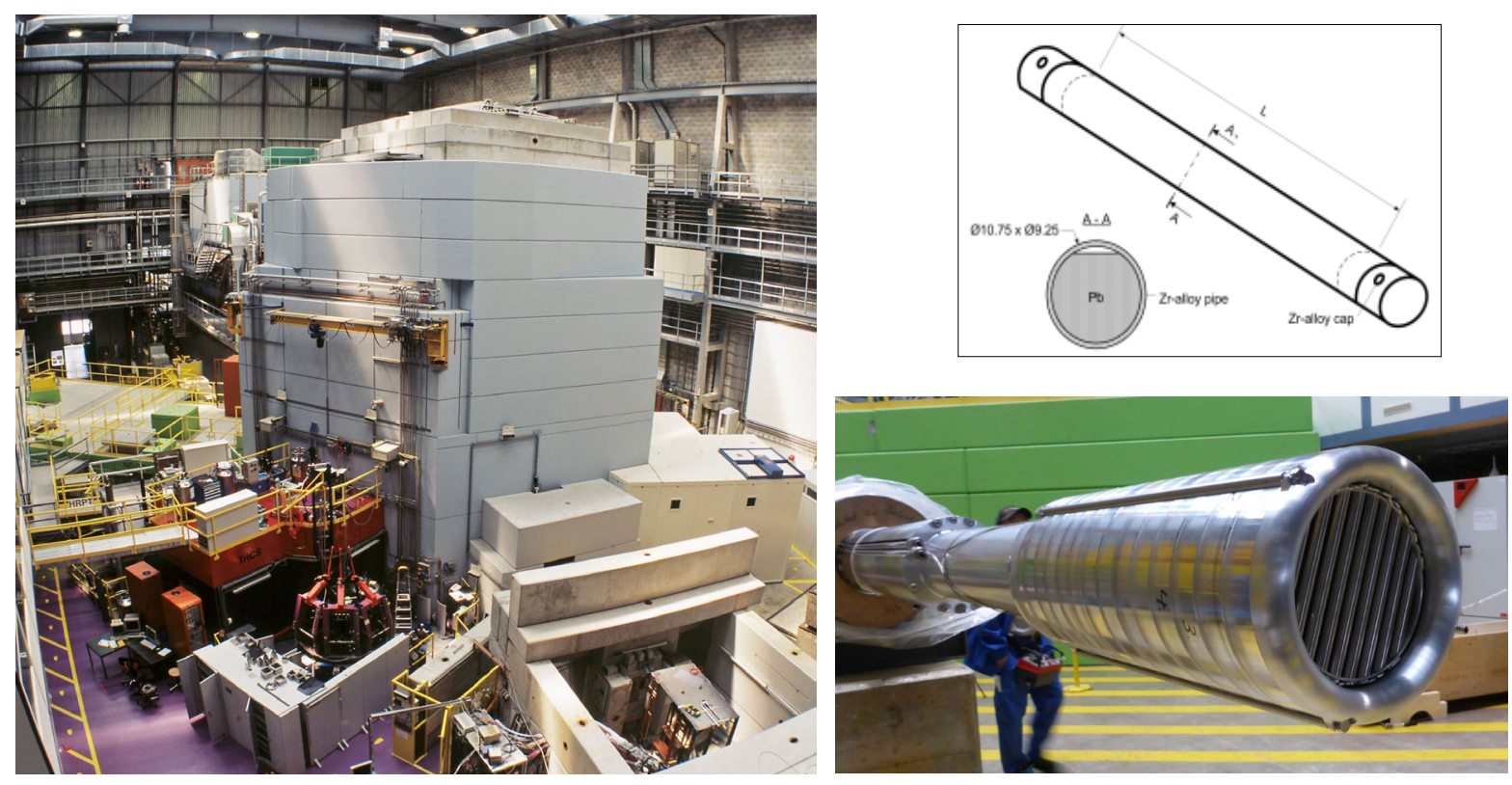

Fig. 1: $\quad$ The spallation neutron source SINQ at PSI (left) and its lead rod target (right); the diameter is approximately $25 \mathrm{~cm}$ only, active length $60 \mathrm{~cm}$. It is positioned vertically in the source center while the proton beam is injected from the bottom

\section{In-situ study of the melting/solidification process (inactive) of target rods}

Standard target rods were used for a study of the lead distribution during cycling above and below the melting point by means of an external heating device using a hot air according to a thermal cycling regime given in Fig. 2. At the same time, neutron images were taken with a common setup with a digital neutron imaging detector (scintillator-camera combination) at the NEUTRA beamline [5]. In order to tolerate the volume expansion during melting, the initial lead filling of Zircaloy cladding tubes was about 50\%.

Some characteristic results are shown in Fig. 3: the sample before cycling and after 357 and 658 cycles, respectively. 


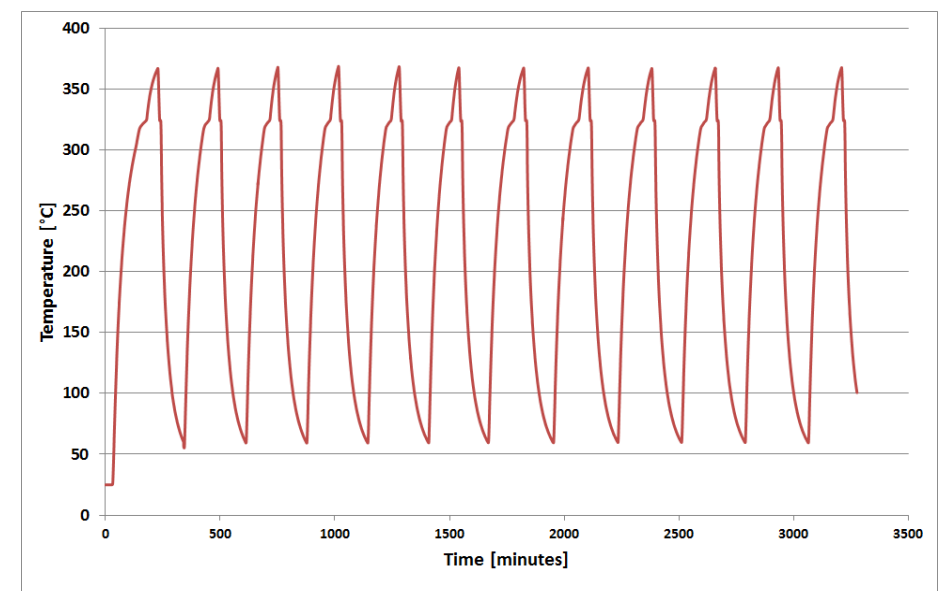

Fig. 2: Temperature evolution at the central point of the target rod shown in Fig. 1 on the right side.

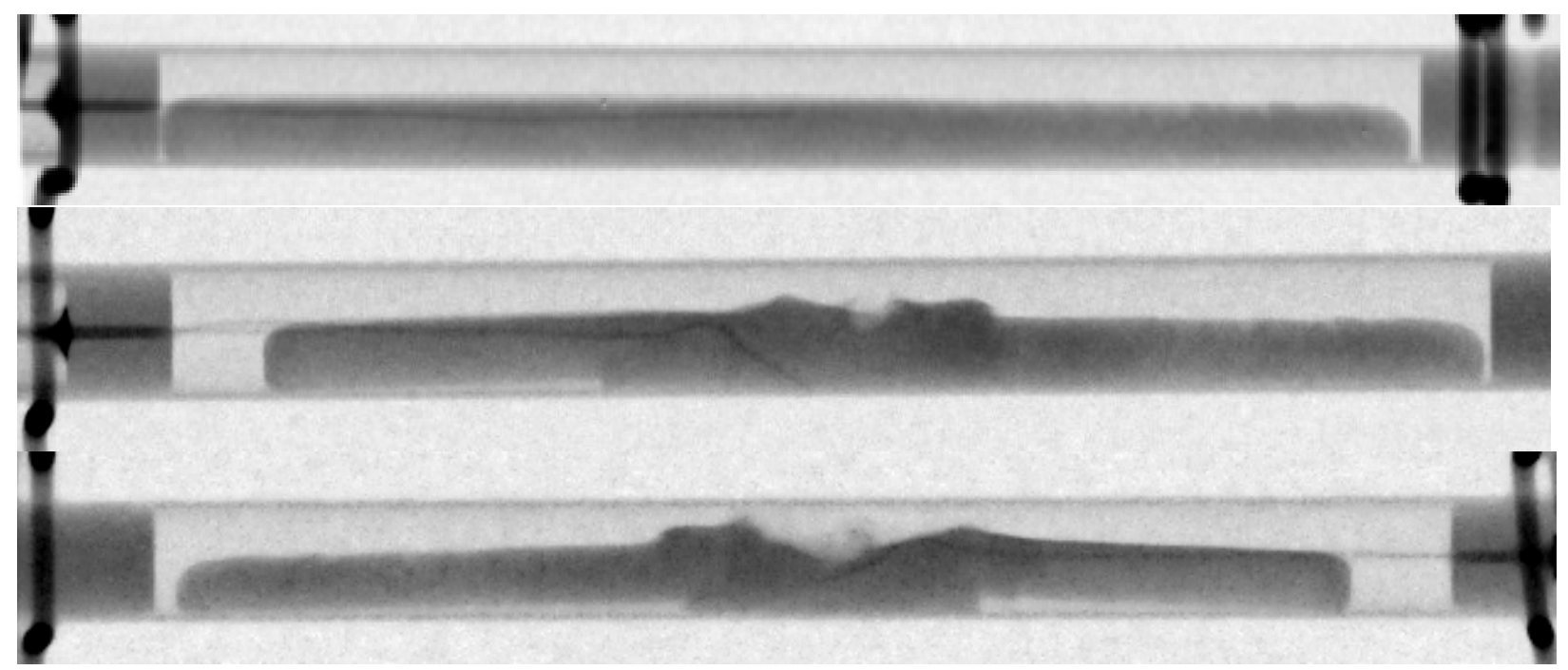

Fig. 3: $\quad$ Dummy test rods with 50\% lead filling in the virgin state (top); the same sample after 357 and 658 thermal cycles, respectively. A thermocouple is used to determine the temperature very precisely (see the data in Fig. 1)

As images shown in Figure 3, prove the lead distribution within Zircaloy cladding tube changes without any mechanical loading. This is particularly apparent in the central part where melting and freezing takes place all time during the cycling. The external parts of lead stay solid, but a certain amount is redistributed towards the center and increases the lead amount there. Because the filling of the investigated rods was only $50 \%$ in volume no risk for cladding damage occurred. However, the real filling in the target rods is $90 \%$ - and some cladding interaction might happen.

Therefore, further, more realistic tests are currently under preparation.

\section{Inspection of hot target rods after «normal» long-term proton exposure (active samples)}

The SINQ target rods are designed for a long-term operation of about 2 years with proton exposure of about $1.5 \mathrm{~mA}$, corresponding to an accumulated proton current of more than 6 Ah. However, there is no material study possible during the operation cycle before the target is exchanged, removed therefore and investigated. One of the non-destructive inspections has been routinely done with a special neutron imaging technique, based on avoidance of the gamma background from the highly activated target material. It is based on the activation of Dy inside 
imaging plates and has to be used in a dedicated regime [6]. Furthermore, the target rod samples have to be extracted under hot-lab conditions and can be only transported to SINQ within a well shielded container. The same container is later be used to manipulate the sample in the beam and to position it towards the Dy-doped imaging plate.
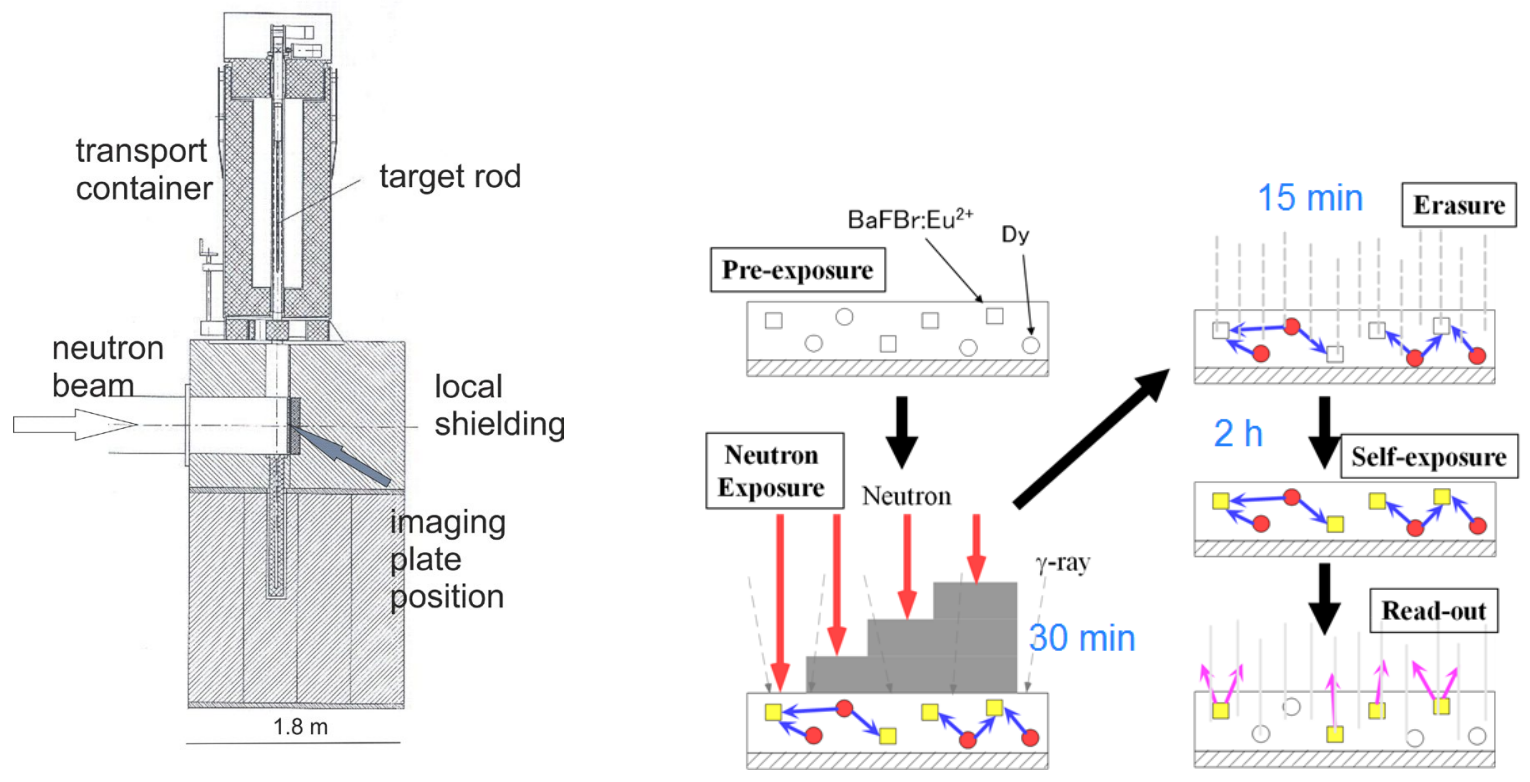

Fig. 4: $\quad$ Setup for the shielded environment for the study of highly-activated samples (left); procedure for using Dy-doped imaging plates to study the neutron induced effects onlywithdrawal of the gamma-background

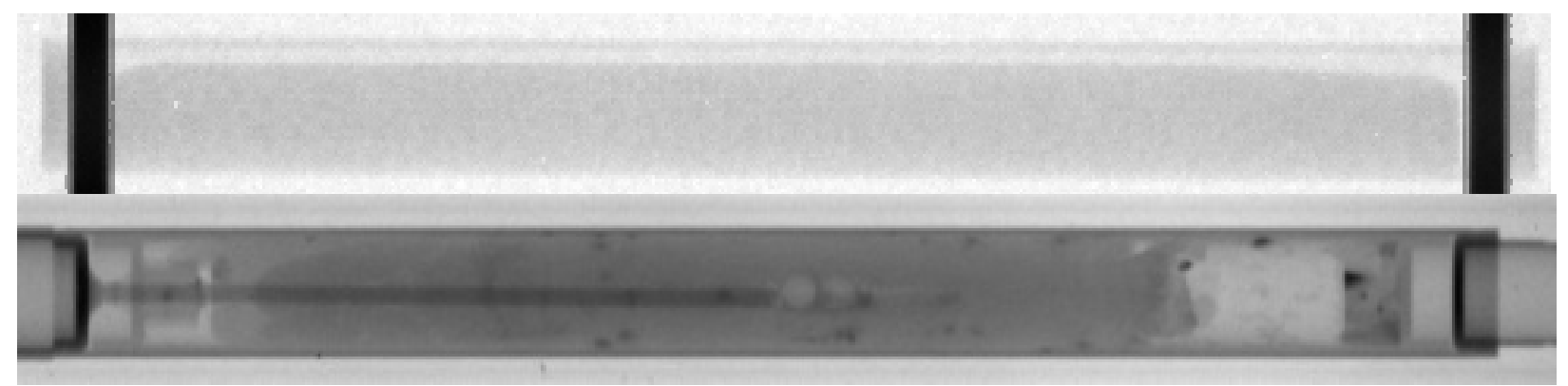

Fig. 5: $\quad$ The "virgin" $\quad \mathrm{Pb}$ rod (top) is quite transparent and uniform - a gap at the upper surface of the rod is visible due to the filling with only $90 \%$ of the volume; (bottom) a long-term exposed rod, containing a central thermal couple, is shown below. The neutron contrast is increased due to the accumulation of highly absorbingly spallation products, such as mercury.

\section{Investigation of target rods after damage (active)}

A target rod failure occurred in SINQ-target No. 8 (operated during 12 month in 2009 and 2010, exposed to 12.15 Ah proton beam). One of the Zircaloy rods failed and lead was thus accidentally released into the coolant flow. The operation of SINQ had to be stopped and the reasons of this failure be assessed.

One of the applied methods was neutron imaging with the option of the suppression of gamma radiation by the Dy-doped imaging plates (as described above). The Figure 6 shows the results together with the photo of the damaged target rod with its long crack in the middle. The neutron imaging data clearly show remaining parts of the lead filling at the edges, fitting well at the right side, but moved to the center on the left side. Some residual material is found diluted in the central part. With the help of the digital information of the image data and the known geometry 
of the rod, it was possible to determine the missing part of lead in the damaged rod. Quantitatively, 38.2 volume per cents of the lead, corresponding to $30.2 \mathrm{~g}$ was missed in the damaged rod and was hence distributed into the coolant circuit.
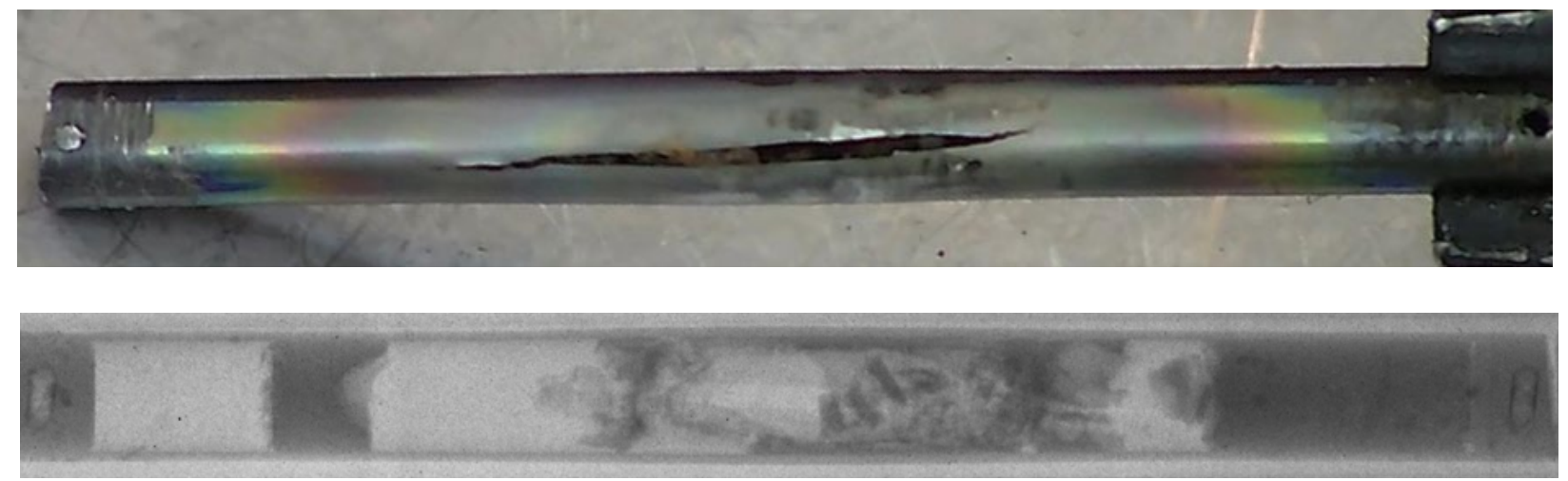

Fig. 6: $\quad$ Photo and neutron image of a broken target rod with lead filling; some of the material is missing and lost by the gap in the cladding

Fortunately, such target rod failures happened very seldom during the 20 years of SINQ operation. However, in order to avoid such target rod failures, it is important to study the material behavior BEFORE some damage happens. Next to the volume expansion of $\mathrm{Pb}$, the hydrogen accumulation in the $\mathrm{Zr}$ cladding during proton exposure has to be seen as a risk because it is accompanied by the risk to embrittlement. Because hydrogen provides a high contrast in neutron imaging data, already small amounts can be defected efficiently.

\section{Summary}

The herewith described neutron imaging techniques were found very useful for non-destructive investigations of target structures containing $\mathrm{Pb}$ and $\mathrm{Zr}$. Both materials are rather transparent for neutrons and very little material changes can be observed sensitively. In the future, the high sensitivity of neutron imaging w.r.t. hydrogen determination will be exploited by means of a high resolution setup, the neutron microscope [8], for the distribution analysis of hydrides.

\section{References}

[1] https://www.psi.ch/science/large-research-facilities

[2] https://www.psi.ch/sinq/sinq

[3] https://www.psi.ch/ucn/ucn-source

[4] https://www.psi.ch/sinq/neutra/sample-environment

[5] https://www.psi.ch/sinq/neutra/

[6] M. Tamaki et al., Dy-IP characterization and its application for experimental neutron radiography tests under realistic conditions, Nuclear Instruments and Methods in Physics Research A 542 (2005) 320-323. https://doi.org/10.1016/j.nima.2005.01.156

[7] W. Gong et al, J. of Nucl. Materials, 2018, submitted.

[8] P. Trtik et al., Improving the Spatial Resolution of Neutron Imaging at Paul Scherrer Institut The Neutron Microscope Project, Physics Procedia 69 ( 2015 ) 169 - 176, https://doi.org/10.1016/j.phpro.2015.07.024 